\title{
In Vitro Two-Dimensional Echocardiographic Imaging of a Stented Porcine Bioprosthetic Valve: The Bent Strut Artifact
}

\author{
David S. Bach, M.D. \\ Department of Internal Medicine, Division of Cardiovascular Medicine, University of Michigan, \\ Ann Arbor, Michigan
}

Background: Echocardiographic imaging of a stented valve bioprosthesis can reveal apparent inward deflection of one or more struts. It could be assumed that this finding is related to actual strut distortion as opposed to an artifact of off-axis imaging. Objective: To determine whether normal (nondistorted) bioprosthetic struts can appear by artifact to be bent inward on two-dimensional echocardiographic imaging. Methods: A production-quality porcine bioprosthetic aortic valve was imaged in vitro using standard two-dimensional echocardiographic techniques. Apparent strut distortion on echocardiographic imaging was investigated relative to prosthesis orientation to the transducer. Results: The appearance of inward strut distortion was produced when two of three struts were simultaneously imaged, including imaging in an off-axis long axis orientation and from above or below the prosthesis. Conclusion: Apparent inward distortion of bioprosthetic struts can be simulated in vitro using a normal, nondistorted valve, and is common if two struts are simultaneously imaged. A finding of inward distortion of strut tips on in vivo imaging should be used with caution, since the finding may not be representative of actual strut anatomy. (ECHOCARDIOGRAPHY, Volume 26, January 2009)

aortic valve prosthesis, mitral valve prosthesis, echocardiography

Two-dimensional echocardiography with Doppler is a reliable tool for the assessment of native and prosthetic valve anatomy and function. The use of multiple imaging planes allows the experienced operator to mentally reconstruct the shape and characteristics of three-dimensional objects. However, irregularly shaped objects and objects uncommonly encountered could be associated with less reliable interpretation.

Stented bioprostheses contain a tricuspid biological valve with an associated sewing cuff and three supporting struts extending perpendicular to the plane of the sewing cuff, which serve to support the three commissural posts; echocardiographic imaging of a bioprosthesis should include assessment of the sewing cuff, struts, and cusps. ${ }^{1}$ Known artifacts associated with echocardiographic imaging of prosthetic

Address for correspondence and reprint requests: David S. Bach, M.D., CVC Room 2147, SPC 5853, 1500 E. Medical Center Drive, Ann Arbor, MI 48109-5853. Fax: 734-6153025; E-mail: dbach@umich.edu valves relate to the reflective properties of prosthetic materials used in the prostheses, and include reverberation artifact and sidelobe artifact. ${ }^{1}$ Apparent inward deflection of the struts can be observed on routine transthoracic and transesophageal echocardiographic imaging (Fig. 1), suggesting malposition of the struts. Some bioprostheses are designed to be implanted with the struts temporarily deflected inward using an associated "cinch" mechanism, intended to prevent suture looping and entanglement around the struts. ${ }^{2}$ The echocardiographic finding of nonparallel orientation of the valve struts later after implantation could suggest incomplete return of the struts to their normal, neutral, position, and has been recently cited as a potential mechanism for increased transvalvular pressure gradients after bioprosthetic aortic valve implantation. ${ }^{3}$ The purpose of the present study was to define whether the echocardiographic appearance of inward deflection of bioprosthesis struts is indicative of strut malposition or other valve distortion. 


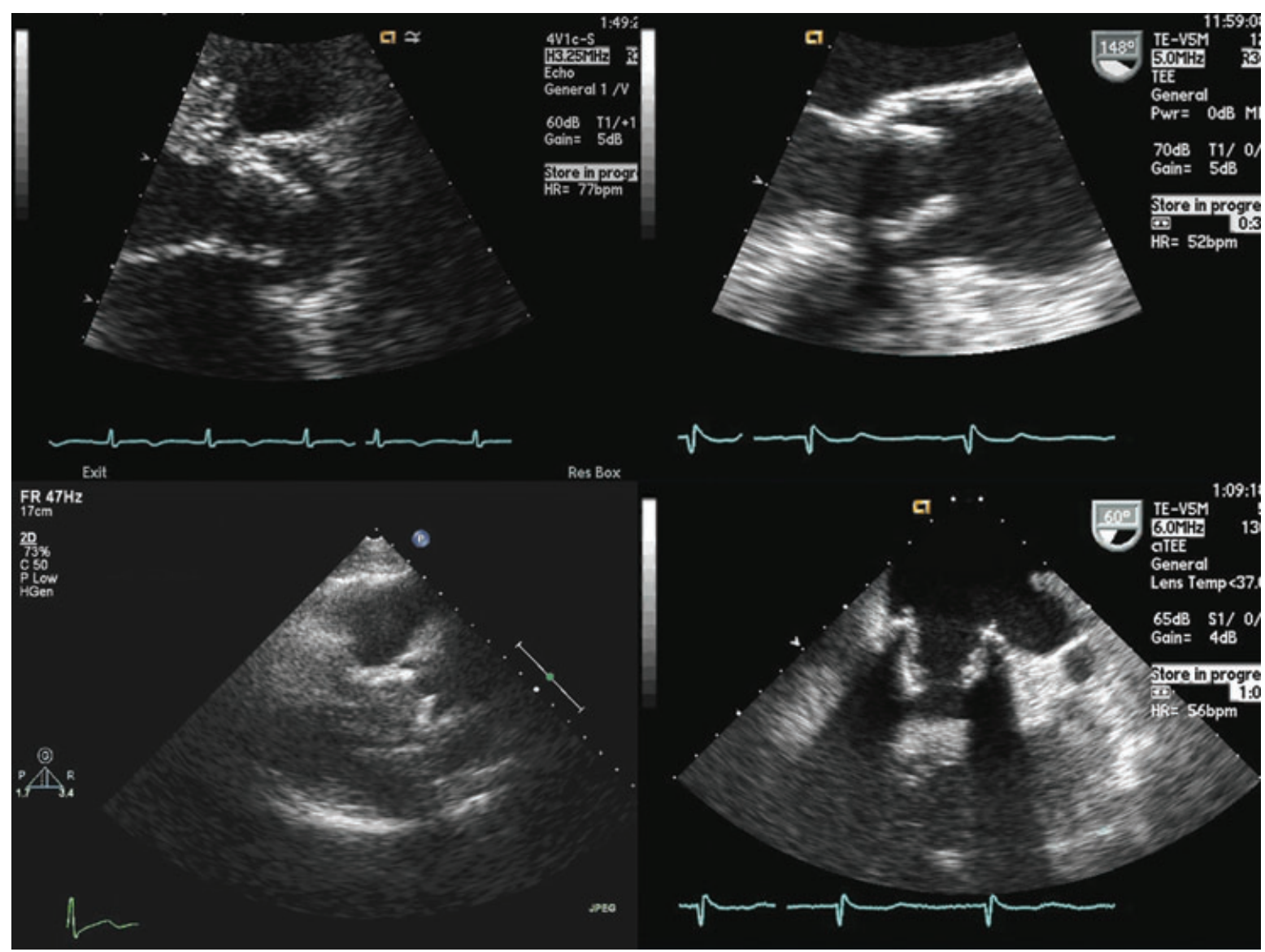

Figure 1. Clinical echocardiographic images revealing apparent inward deflection (from sewing cuff to commissural posts) of various bioprostheses. A. Mosaic (Medtronic, Inc., Minneapolis, MN, USA) valve in aortic position, transthoracic imaging. B. Mosaic valve in aortic position, transesophageal imaging. C. Epic (St Jude Medical, Inc., St Paul, MN, USA) valve in aortic position, transthoracic imaging. D. Mosaic valve in mitral position, transesophageal imaging.

\section{Methods}

A production-quality, clinical-grade $23 \mathrm{~mm}$ Mosaic standard porcine aortic bioprosthesis (Medtronic, Inc., Minneapolis, MN, USA) suitable for human implantation was obtained from the manufacturer. The valve was removed from its packaging and sterile container. Sutures holding the valve to its associated cinch mechanism were removed, and the valve was not further manipulated. For purposes of imaging, the valve was held using a small hemostat attached to a single point on the valve sewing cuff. The hemostat "bite" was oriented perpendicular to the short axis of the sewing cuff, in the same direction as a suture would be used during valve implantation. Because of this orientation and the single point of attachment, there was no potential for distortion of the valve orifice or struts.

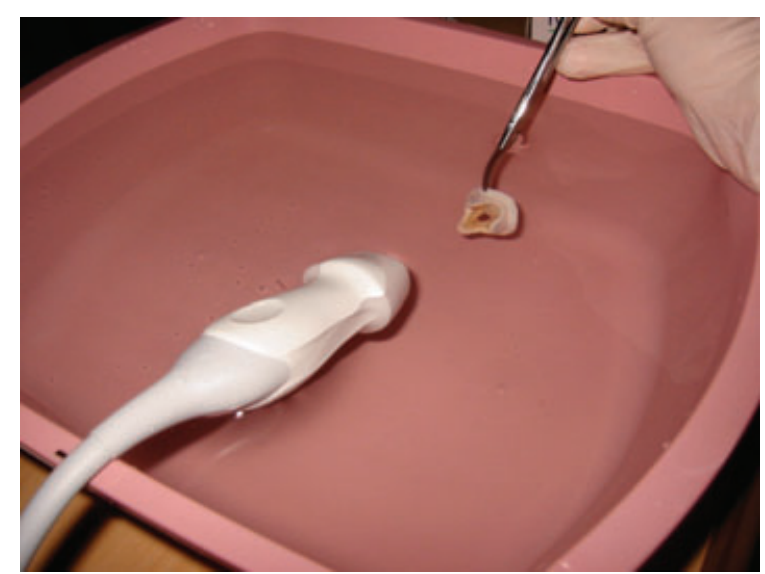

Figure 2. Imaging methodology. Production-quality porcine bioprosthesis was imaged in vitro using standard two-dimensional echocardiographic equipment. 


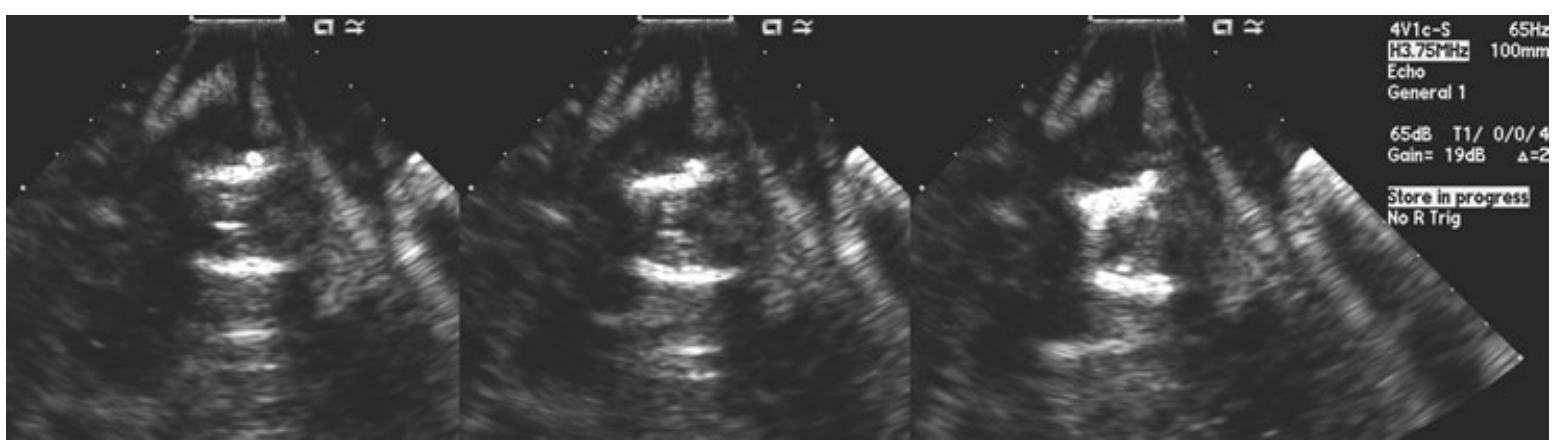

Figure 3. Serial long-axis views of bioprosthesis moved through a two-dimensional transducer beam without other changes in valve orientation. Apparent strut distortion is noted (right panel) when valve is imaged off its midline such that two of the three struts are shown (sewing cufflinflow edge on right; strut tips/outflow on left in each image).

The valve was submerged in a $\sim 6$ liter water bath that was filled with room-temperature tap water and allowed to stand for 1 hour before imaging. Echocardiographic imaging was performed using an Acuson Sequoia C512 (Siemens US, Malvern, PA, USA) and Siemens 4V1c-S transthoracic transducer at $3.75 \mathrm{MHz}$. The transducer was partially submerged in the water bath and held in a fixed position; change in orientation between the transducer and the valve prosthesis was achieved through controlled movement of the hemostat holding the prosthesis (Fig. 2). The bioprosthesis was imaged in multiple axes. Images were digitally acquired and reproduced with cropping but no other image alteration.

\section{Results}

On visual inspection, the valve struts were perpendicular to the sewing cuff, with neither distortion nor inward deflection. When imaged

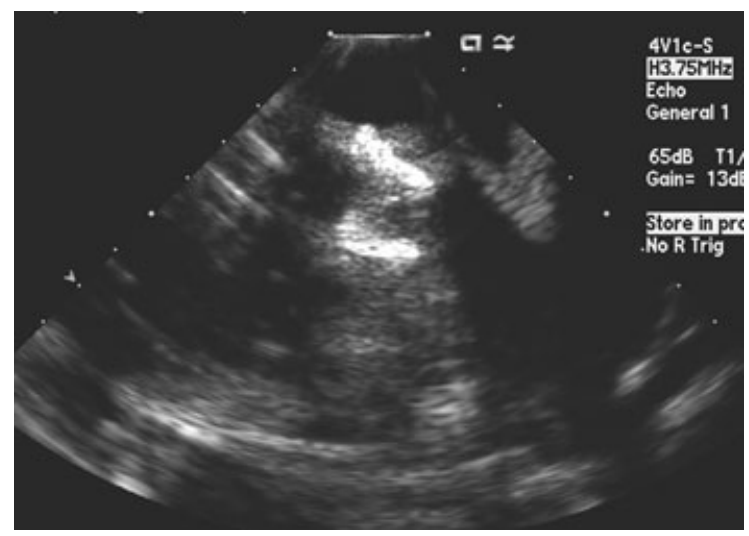

Figure 4. Bioprosthesis imaged from slightly above its inflow aspect (sewing cufflinflow edge on left; strut tips/ outflow on right). from the side, simulating a long-axis view of the valve, a variety of images suggesting apparently different strut patterns were produced. Figure 3 shows a series of images in which the bioprosthesis is slowly moved through the twodimensional ultrasound beam, such that the beam intersects different portions of the prosthesis in its long axis, but without any other

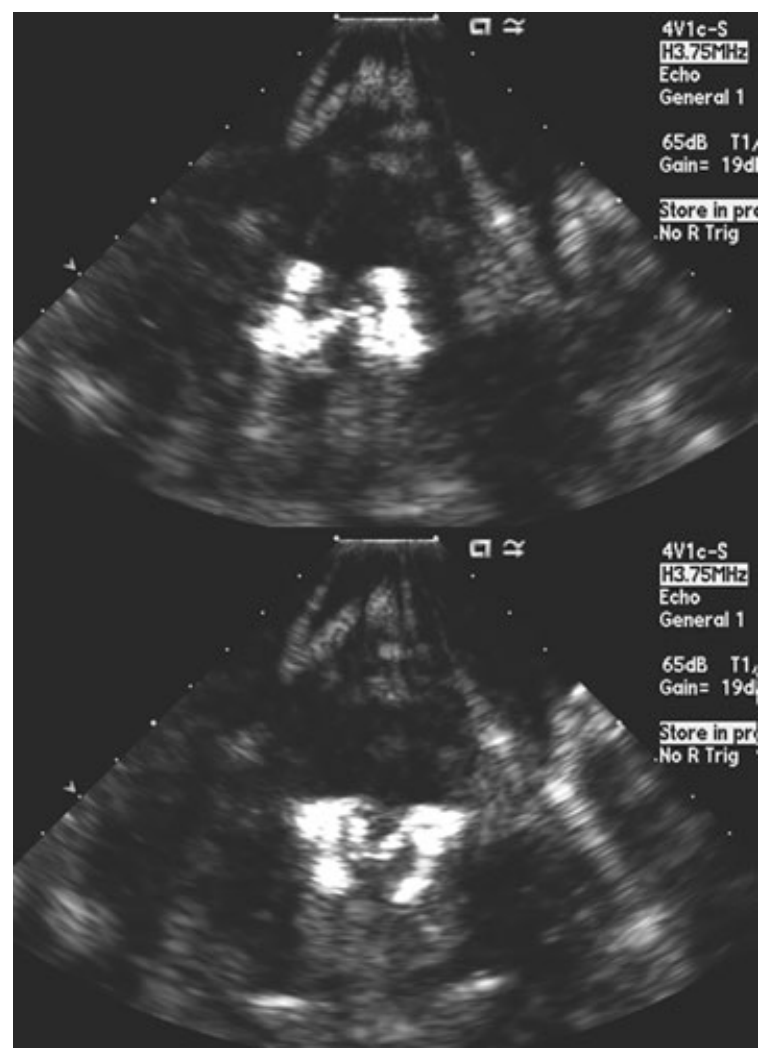

Figure 5. Bioprosthesis imaged from directly above its outflow aspect (upper panel) and inflow aspect (lower panel). 
Figure 6. Beat-to-beat variability of apparent strut orientation on clinical transesophageal echocardiogram of porcine bioprosthesis in aortic position. The on-axis longaxis view (upper panel, depicting one strut and sewing cuff midway between other two struts) suggests normal strut orientation, whereas simultaneous imaging of two struts suggests inward strut distortion (lower panel).

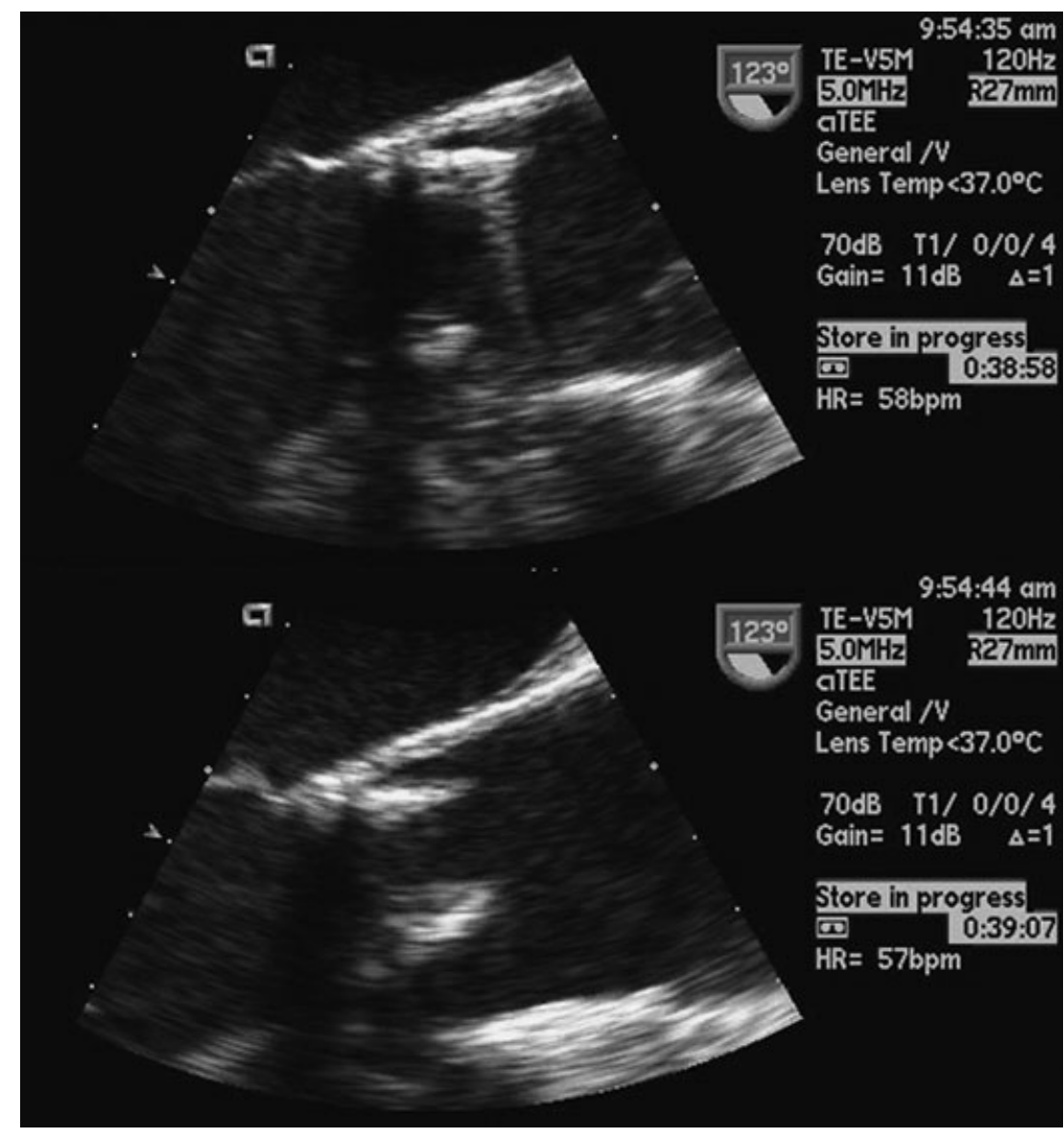

change in valve orientation. Images that include clear display of two struts (right panel) were associated with apparent strut distortion. Figure 4 shows the same valve imaged from slightly above its inflow aspect. Figure 5 shows the same prosthesis imaged from directly above and below.

\section{Discussion}

Standard two-dimensional echocardiography relies on imaging three-dimensional objects in multiple two-dimensional planes, allowing an experienced operator to mentally reconstruct the three-dimensional object. This is most reliably accomplished when the imaged object is symmetrical in shape or conforms to known geometry. A stented bioprosthesis contains a tricuspid biological valve with an associated prosthetic sewing cuff and three supporting struts extending perpendicular to the plane of the sewing cuff. When imaged in two dimensions, simultaneous depiction of two struts that are not parallel to each other could lead to the con- clusion that the struts are not parallel, and a clinical interpretation that one or both struts are distorted from their normal position. However, despite concern that this could represent a clinical problem, ${ }^{3}$ there has been no known documented pathologic confirmation of strut distortion associated with this in vivo echocardiographic finding.

The struts of a stented bioprosthesis are never $180^{\circ}$ apart; depending on the specific bioprosthesis, they occur approximately (but not necessarily exactly) $120^{\circ}$ around the sewing cuff. As such, any two-dimensional image that includes two struts is by definition off axis, since an on-axis long-axis view through one strut and through the center of the bioprosthetic orifice would bisect the space between the two remaining struts.

In this study, a clinical-grade stented porcine bioprosthesis was imaged in vitro. By visual inspection before, during, and after imaging, the struts were normal and undistorted. As such, any echocardiographic image that displayed nonparallel struts was by definition specious 
and related to artifact. In this model, apparent inward distortion of the struts from sewing cuff toward strut tips was observed in several orientations, all of which involved simultaneous imaging of two struts. When the valve was imaged in a plane perpendicular to its long axis (equivalent to in vivo long-axis imaging in which strut distortion might be interrogated), simultaneous imaging of two struts resulted in images suggesting an apparent inward distortion of the struts (Fig. 3). The same pattern was apparent when two struts were simultaneously imaged from slightly or directly above or below the valve (Figs. 4 and 5).

Clinically, in vivo imaging of a stented bioprosthesis can be associated with significant beat-to-beat variability of apparent strut orientation (Fig. 6). When imaged in an on-axis long-axis view (depicting one strut and the valve sewing cuff midway between the other two struts), strut orientation appears normal. However, inward strut distortion is suggested when two struts are simultaneously imaged.

In conclusion, apparent inward distortion of a bioprosthesis strut can be simulated in vitro us- ing a normal, nondistorted valve, and appears to be related to simultaneous (off-axis) imaging of two of the three struts. Side-lobe artifact likely plays a role in the appearance of a second strut in many echocardiographic views. A finding of inward distortion of one or more struts on in vivo imaging of a bioprosthesis should be used with caution, since the finding may be artifactual and not representative of actual strut anatomy.

Acknowledgment: Material support for this research was provided by Medtronic Heart Valves, Minneapolis, MN, USA.

\section{References}

1. Otto CM: Prosthetic valves. In Otto CM (ed.): Textbook of Clinical Echocardiography, 3rd Ed. Philadelphia, PA, Elsevier Saunders, 2004, pp. 355-381.

2. http://www.ctsnet.org/file/vendors/795/pdf/cinch_bro72.pdf. Accessed February 18, 2008.

3. Buck T, Janosi A, Wendt A, et al: Echocardiographic detection of high pressure gradients and small orifice areas in a novel aortic valve bioprosthesis necessitating termination of utilization (abstract). Eur Heart $J$ 2007;28(Suppl):824. 